\title{
Robust stability of linear neutral systems with nonlinear parameter perturbations
}

\author{
Q.-L. Han and L. Yu
}

\begin{abstract}
The robust stability of uncertain linear neutral systems with time-varying discrete and neutral delays is investigated. The uncertainties under consideration are nonlinear time-varying parameter perturbations and norm-bounded uncertainties, respectively. Both delay-dependent and delay-derivative-dependent stability criteria are proposed and are formulated in the form of linear matrix inequalities. The presented results contain some existing results as their special cases. Numerical examples are also given to indicate significant improvements over existing results.
\end{abstract}

\section{Nomenclature}

\begin{tabular}{|c|c|}
\hline $\mathbb{R}$ & : the set of real numbers \\
\hline $\mathbb{R}^{n}$ & $\begin{array}{l}\text { the set of real } n \text {-dimensional } \\
\text { vectors }\end{array}$ \\
\hline $\mathbb{R}^{n \times m}$ & $\begin{array}{l}: \text { the set of real } n \times m \text {-dimensional } \\
\text { matrices }\end{array}$ \\
\hline $\boldsymbol{W}^{T}$ & : transpose of matrix $\boldsymbol{W}$ \\
\hline $\boldsymbol{W}>0(\boldsymbol{W}<0)$ & $\begin{array}{l}: \boldsymbol{W} \text { is a symmetric positive } \\
\text { (negative) definite matrix }\end{array}$ \\
\hline $\boldsymbol{W} \geq 0(\boldsymbol{W} \leq 0)$ & $\begin{array}{l}: \boldsymbol{W} \text { is a symmetric positive } \\
\text { (negative) semi-definite matrix }\end{array}$ \\
\hline$\lambda_{\max }(\boldsymbol{W})\left(\lambda_{\min }(\boldsymbol{W})\right.$ & $\begin{array}{l}\text { : the maximum (minimum) eigen- } \\
\text { value of a symmetric matrix } \boldsymbol{W}\end{array}$ \\
\hline$\|\boldsymbol{M}\|$ & $\begin{array}{l}\text { the Euclidean vector norm or its } \\
\text { induced matrix 2-norm of a vector } \\
\text { or a matrix } \boldsymbol{M}\end{array}$ \\
\hline 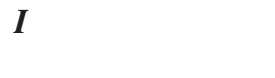 & $\begin{array}{l}\text { : identity matrix of appropriate } \\
\text { dimensions }\end{array}$ \\
\hline
\end{tabular}

\section{Introduction}

The problem of the stability of delay-differential neutral systems has received considerable attention in the last two decades; for example, [1]. Practical examples of neutral systems include distributed networks containing lossless transmission lines [2], and population ecology [3]. Depending on whether or not the stability criterion contains delay information, it can be divided into one of two categories; namely, either a delay-independent stability criteria $[4,5]$ or a delay-dependent stability criteria [6-10]. However, to date only neutral systems with a constant neutral delay have been considered.

In recent years, the problem of the robust stability of retarded systems with nonlinear parameter perturbations has also received considerable attention. In [11], for example, delay-independent and delay-dependent stability criteria

\section{(C) IEE, 2004}

IEE Proceedings online no. 20040785

doi: 10.1049/ip-cta:20040785

Paper first received 13th January and in revised form 14th May 2004

Q.-L. Han is with the Faculty of Informatics and Communication, Central Queensland University, Rockhampton, Qld 4702, Australia

L. Yu is with the Department of Automation, Zhejiang University of Technology, Hangzhou 310032, People's Republic of China are obtained by using matrix properties and a comparison theorem. In [12], based on matrix measure, matrix norm and a decomposition technique, two stability criteria are derived. The results in [11] and [12] are very conservative since they required the matrix measure to be negative. In [13], a model transformation technique is used to transform a system with a discrete delay to a system with a distributed delay, and delay-dependent stability criteria are obtained by using a Lyapunov-Krasovskii functional approach. Although the results in [13] are less conservative than many existing ones, they are still conservative since the model transformation introduces additional dynamics as discussed in [14]. In [15], based on a descriptor model transformation [9] and the decomposition of a discrete delay term matrix, the robust stability of uncertain systems with a single time-varying discrete delay is investigated by applying an integral inequality as compared to the bounding of cross-terms used in [16]. Numerical examples show that the results obtained in [15] are less conservative than existing ones in the literature. To the best of our knowledge, the problem of the robust stability of neutral systems with nonlinear parameter perturbations has not been addressed in the case of a time-varying neutral delay.

We will now investigate the robust stability of uncertain neutral systems using the Lyapunov-Krasovskii functional approach. We will consider both nonlinear parameter perturbations and norm-bounded uncertainties. The delays under considerations will include time-varying discrete and neutral delays. We will consider the robust stability problem in terms of symmetric positive-definite matrices. Both delay-dependent and delay-derivative-dependent stability criteria will be proposed and formulated in the form of linear matrix inequalities (LMIs), which can be effectively solved using interior-point optimisation algorithms [17].

In this work, a delay-dependent stability criterion for linear systems with a time-varying delay means that the criterion itself contains information on both the bound and delay-derivative bound of the time-varying delay whereas a delay-derivative-dependent criterion only contains information on the delay-derivative bound of the time-varying delay. For the case of a constant time-delay, the delay-derivative-dependent criterion reduces to delayindependent one.

\section{Problem statement}

Consider the following linear neutral system with timevarying discrete and neutral delays: 


$$
\begin{aligned}
\dot{\boldsymbol{x}}(t)= & \boldsymbol{A} \boldsymbol{x}(t)+\boldsymbol{B} \boldsymbol{x}(t-r(t))+\boldsymbol{C} \dot{\boldsymbol{x}}(t-\tau(t)) \\
& +\boldsymbol{f}(\boldsymbol{x}(t), t)+\boldsymbol{g}(\boldsymbol{x}(t-r(t)), t)+\boldsymbol{h}(\dot{\boldsymbol{x}}(t-\tau(t)), t)
\end{aligned}
$$

where $\boldsymbol{x}(t) \in \mathbb{R}^{n}$ is the state, $\boldsymbol{A} \in \mathbb{R}^{n \times n}, \boldsymbol{B} \in \mathbb{R}^{n \times n}$ and $\boldsymbol{C} \in \mathbb{R}^{n \times n}$ are constant matrices. The time-varying vectorvalued functions $\boldsymbol{f}(\boldsymbol{x}(t), t) \in \mathbb{R}^{n}, \boldsymbol{g}(\boldsymbol{x}(t-r(t)), t) \in \mathbb{R}^{n}$ and $\boldsymbol{h}(\dot{\boldsymbol{x}}(t-\tau(t)), t) \in \mathbb{R}^{n}$ are unknown and represent the parameter perturbations with respect to the current state $\boldsymbol{x}(t)$ and delayed state $\boldsymbol{x}(t-r(t))$ and $\dot{\boldsymbol{x}}(t-\tau(t))$ of the system, respectively. They satisfy that $\boldsymbol{f}(0, t)=0, \boldsymbol{g}(0, t)=$ 0 and $\boldsymbol{h}(0, t)=0$. The delay $r(t)$ is a time-varying discrete delay and $\tau(t)$ is a time-varying neutral delay, which satisfy:

$$
0 \leq r(t) \leq r_{\mathrm{M}} \quad \dot{r}(t) \leq r_{\mathrm{d}} \quad 0 \leq \tau(t) \leq \tau_{\mathrm{M}} \quad \dot{\tau}(t) \leq \tau_{\mathrm{d}}
$$

where $r_{\mathrm{M}}, r_{\mathrm{d}}, \tau_{\mathrm{M}}$ and $\tau_{\mathrm{d}}$ are constants, and $0 \leq r_{\mathrm{d}}<1$ and $0 \leq \tau_{\mathrm{d}}<1$.

The initial condition of system (1) is given by:

$$
\begin{aligned}
\boldsymbol{x}\left(t_{0}+\theta\right)=\boldsymbol{\varphi}(\theta) & \dot{\boldsymbol{x}}\left(t_{0}+\theta\right)=\dot{\boldsymbol{\varphi}}(\theta) \\
& \forall \theta \in\left[-\max \left\{r_{\mathrm{M}}, \tau_{\mathrm{M}}\right\}, 0\right]
\end{aligned}
$$

where $\boldsymbol{\varphi}(\cdot)$ is a vector-valued initial function.

The aim of this work is to formulate some practically computable criteria to check the stability of the system described by (1)-(3).

\section{Nonlinear time-varying parameter perturbation}

In this Section, we assume that $\boldsymbol{f}(\boldsymbol{x}(t), t), \boldsymbol{g}(\boldsymbol{x}(t-h(t)), t)$ and $\boldsymbol{h}(\dot{\boldsymbol{x}}(t-\tau(t)), t)$ represent the nonlinear parameter timevarying perturbations of system (1) which satisfy that:

$$
\begin{gathered}
\|\boldsymbol{f}(\boldsymbol{x}(t), t)\| \leq \alpha\|\boldsymbol{x}(t)\| \\
\|\boldsymbol{g}(\boldsymbol{x}(t-r(t)), t)\| \leq \beta\|\boldsymbol{x}(t-r(t))\| \\
\|\boldsymbol{h}(\dot{\boldsymbol{x}}(t-\tau(t)), t)\| \leq \gamma\|\dot{\boldsymbol{x}}(t-\tau(t))\|
\end{gathered}
$$

where $\alpha \geq 0, \beta \geq 0$ and $\gamma \geq 0$ are given constants.

Constraint (4) can be rewritten as:

$$
\begin{gathered}
\boldsymbol{f}^{T}(\boldsymbol{x}(t), t) \boldsymbol{f}(\boldsymbol{x}(t), t) \leq \alpha^{2} \boldsymbol{x}^{T}(t) \boldsymbol{x}(t) \\
\boldsymbol{g}^{T}(\boldsymbol{x}(t-r(t)), t) \boldsymbol{g}(\boldsymbol{x}(t-r(t)), t) \\
\quad \leq \beta^{2} \boldsymbol{x}^{T}(t-r(t)) \boldsymbol{x}(t-r(t)) \\
\boldsymbol{h}^{T}(\dot{\boldsymbol{x}}(t-\tau(t)), t) \boldsymbol{h}(\dot{\boldsymbol{x}}(t-\tau(t)), t) \\
\leq \gamma^{2} \dot{\boldsymbol{x}}^{T}(t-\tau(t)) \dot{\boldsymbol{x}}(t-\tau(t))
\end{gathered}
$$

For the robust stability of system (1)-(3), with uncertainty (4), we have the following delay-dependent stability result.

Proposition 1: The system described by (1)-(3), with uncertainty described by (4) is asymptotically stable if $\|\boldsymbol{C}\|+\gamma<1$ and there exists a real matrix $\boldsymbol{X}$, symmetric positive definite matrices $\boldsymbol{P}, \boldsymbol{R}, \boldsymbol{S}, \boldsymbol{Y}$ and scalars $\varepsilon_{1} \geq 0$, $\varepsilon_{2} \geq 0$ and $\varepsilon_{3} \geq 0$ such that the following LMI holds:

$\left(\begin{array}{cccccc}(1,1) & -\boldsymbol{X}^{T} \boldsymbol{B} & \boldsymbol{P C} & \boldsymbol{P} & \boldsymbol{P} & \boldsymbol{P} \\ -\boldsymbol{B}^{T} \boldsymbol{X} & (2,2) & 0 & 0 & 0 & 0 \\ \boldsymbol{C}^{T} \boldsymbol{P} & 0 & (3,3) & 0 & 0 & 0 \\ \boldsymbol{P} & 0 & 0 & -\varepsilon_{1} \boldsymbol{I} & 0 & 0 \\ \boldsymbol{P} & 0 & 0 & 0 & -\varepsilon_{2} \boldsymbol{I} & 0 \\ \boldsymbol{P} & 0 & 0 & 0 & 0 & -\varepsilon_{3} \boldsymbol{I} \\ \boldsymbol{S} \boldsymbol{A} & \boldsymbol{S} \boldsymbol{B} & \boldsymbol{S C} & \boldsymbol{S} & \boldsymbol{S} & \boldsymbol{S} \\ \boldsymbol{Y B A} & \boldsymbol{Y} \boldsymbol{B} \boldsymbol{B} & \boldsymbol{Y} \boldsymbol{B} \boldsymbol{C} & \boldsymbol{Y} \boldsymbol{B} & \boldsymbol{Y} \boldsymbol{B} & \boldsymbol{Y} \boldsymbol{B} \\ r_{\mathrm{M}}(\boldsymbol{X}+\boldsymbol{P}) & 0 & 0 & 0 & 0 & 0 \\ & \boldsymbol{A}^{T} \boldsymbol{S} & \boldsymbol{A}^{T} \boldsymbol{B}^{T} \boldsymbol{Y} & r_{\mathrm{M}}\left(\boldsymbol{X}^{T}+\boldsymbol{P}\right) \\ & \boldsymbol{B}^{T} \boldsymbol{S} & \boldsymbol{B}^{T} \boldsymbol{B}^{T} \boldsymbol{Y} & 0 \\ & \boldsymbol{C}^{T} \boldsymbol{S} & \boldsymbol{C}^{T} \boldsymbol{B}^{T} \boldsymbol{Y} & 0 \\ & \boldsymbol{S} & \boldsymbol{B}^{T} \boldsymbol{Y} & 0 \\ \boldsymbol{S} & \boldsymbol{B}^{T} \boldsymbol{Y} & 0 \\ \boldsymbol{S} & \boldsymbol{B}^{T} \boldsymbol{Y} & 0 \\ & -\boldsymbol{S} & 0 & 0 \\ & 0 & -\boldsymbol{Y} & 0 \\ & 0 & 0 & -\boldsymbol{Y}\end{array}\right)<0 \quad(6)$

where

$(1,1) \triangleq(\boldsymbol{A}+\boldsymbol{B})^{T} \boldsymbol{P}+\boldsymbol{P}(\boldsymbol{A}+\boldsymbol{B})+\boldsymbol{R}+\boldsymbol{X}^{T} \boldsymbol{B}+\boldsymbol{B}^{T} \boldsymbol{X}+\varepsilon_{1} \alpha^{2} \boldsymbol{I}$

$(2,2) \stackrel{\Delta}{=}-\left(1-r_{\mathrm{d}}\right) \boldsymbol{R}+\varepsilon_{2} \beta^{2} \boldsymbol{I}$

$(3,3) \stackrel{\Delta}{=}-\left(1-\tau_{\mathrm{d}}\right) \boldsymbol{S}+\varepsilon_{3} \gamma^{2} \boldsymbol{I}$

Proof: Choose a Lyapunov-Krasovskii functional candidate for system (1) as:

$$
V(t)=V_{1}(t)+V_{2}(t)+V_{3}(t)+V_{4}(t)
$$

where

$$
\begin{aligned}
& V_{1}(t)=\boldsymbol{x}^{T}(t) \boldsymbol{P} \boldsymbol{x}(t) \\
& V_{2}(t)=\int_{t-r_{\mathrm{M}}}^{t}\left(r_{\mathrm{M}}-t+\xi\right) \dot{\boldsymbol{x}}^{T}(\xi) \boldsymbol{B}^{T} \boldsymbol{Q B} \dot{\boldsymbol{x}}(\xi) d \xi \\
& V_{3}(t)=\int_{t-r(t)}^{t} \boldsymbol{x}^{T}(\xi) \boldsymbol{R} \boldsymbol{x}(\xi) d \xi \\
& V_{4}(t)=\int_{t-\tau(t)}^{t} \dot{\boldsymbol{x}}^{T}(\xi) \boldsymbol{S} \dot{\boldsymbol{x}}(\xi) d \xi
\end{aligned}
$$

where symmetric positive definite matrices $\boldsymbol{P}, \boldsymbol{R}, \boldsymbol{S}, \boldsymbol{Y}$ $\left(=r_{\mathrm{M}} \boldsymbol{Q}\right)$ are solutions of (6).

It is easy to see that:

$$
\begin{aligned}
& \lambda_{\min }(\boldsymbol{P})\|\boldsymbol{x}(t)\|^{2} \\
& \leq V(t) \leq \omega\left(\left[\|\boldsymbol{x}(t)\|^{2}+\int_{-\max \left\{r_{\mathrm{M}}, \tau_{\mathrm{M}}\right\}}^{0}\|\dot{\boldsymbol{x}}(t+\theta)\|^{2} d \theta\right]^{1 / 2}\right)^{2}
\end{aligned}
$$

where $\omega \triangleq \max \left\{\lambda_{\max }(\boldsymbol{P})+2 r_{\mathrm{M}} \lambda_{\max }(\boldsymbol{R}), r_{\mathrm{M}} \lambda_{\max }\left(\boldsymbol{B}^{T} \boldsymbol{Q B}\right)+\right.$ $\left.2 r_{\mathrm{M}}^{2} \lambda_{\max }(\boldsymbol{R})+\lambda_{\max }(\boldsymbol{S})\right\}$.

The derivative of $V(t)$ along the trajectory of system (1) is given by $\dot{V}(t)=\dot{V}_{1}(t)+\dot{V}_{2}(t)+\dot{V}_{3}(t)+\dot{V}_{4}(t)$. To derive discrete-delay-dependent stability condition, which includes the information of the time-delay $r(t)$, one usually uses the fact

IEE Proc.-Control Theory Appl., Vol. 151, No. 5, September 2004 


$$
\boldsymbol{x}(t-r(t))=\boldsymbol{x}(t)-\int_{t-r(t)}^{t} \dot{\boldsymbol{x}}(\xi) d \xi
$$

to transform system (1) to the following one:

$$
\begin{aligned}
\dot{\boldsymbol{x}}(t)= & (\boldsymbol{A}+\boldsymbol{B}) \boldsymbol{x}(t)-\boldsymbol{B} \int_{t-r(t)}^{t} \dot{\boldsymbol{x}}(\xi) d \xi+\boldsymbol{C} \dot{\boldsymbol{x}}(t-\tau(t)) \\
& +\boldsymbol{f}(\boldsymbol{x}(t), t)+\boldsymbol{g}(\boldsymbol{x}(t-r(t)), t)+\boldsymbol{h}(\dot{\boldsymbol{x}}(t-\tau(t)), t)
\end{aligned}
$$

We have

$$
\begin{aligned}
\dot{V}_{1}(t)= & 2 \boldsymbol{x}^{T}(t) \boldsymbol{P}(\boldsymbol{A}+\boldsymbol{B}) \boldsymbol{x}(t)-2 \boldsymbol{x}^{T}(t) \boldsymbol{P} \boldsymbol{B} \int_{t-r(t)}^{t} \dot{\boldsymbol{x}}(\xi) d \xi \\
& +2 \boldsymbol{x}^{T}(t) \boldsymbol{P C} \dot{\boldsymbol{x}}(t-\tau(t))+2 \boldsymbol{x}^{T}(t) \boldsymbol{P} \boldsymbol{f}(\boldsymbol{x}(t), t) \\
& +2 \boldsymbol{x}^{T}(t) \boldsymbol{P} \boldsymbol{g}(\boldsymbol{x}(t-r(t)), t)+2 \boldsymbol{x}^{T}(t) \boldsymbol{P h}(\dot{\boldsymbol{x}}(t-\tau(t)), t)
\end{aligned}
$$

Define $\boldsymbol{a}(\xi)=\boldsymbol{B} \dot{\boldsymbol{x}}(\xi), \boldsymbol{b}(\xi)=\boldsymbol{P} \boldsymbol{x}(t)$ and use lemma 1 in [16] to obtain:

$$
\begin{aligned}
-2 & \boldsymbol{x}^{T}(t) \boldsymbol{P} \boldsymbol{B} \int_{t-r(t)}^{t} \dot{\boldsymbol{x}}(\xi) d \xi \\
\leq & r_{\mathrm{M}} \boldsymbol{x}^{T}(t) \boldsymbol{P}\left(\boldsymbol{M}^{T} \boldsymbol{Q}+\boldsymbol{I}\right) \boldsymbol{Q}^{-1}(\boldsymbol{Q M}+\boldsymbol{I}) \boldsymbol{P} \boldsymbol{x}(t) \\
& +2 \boldsymbol{x}^{T}(t) \boldsymbol{P} \boldsymbol{M}^{T} \boldsymbol{Q B} \int_{t-r(t)}^{t} \dot{\boldsymbol{x}}(\xi) d \xi+\int_{t-r(t)}^{t} \dot{\boldsymbol{x}}^{T}(\xi) \boldsymbol{B}^{T} \boldsymbol{Q} \boldsymbol{B} \dot{\boldsymbol{x}}(\xi) d \xi
\end{aligned}
$$

Let $\boldsymbol{X}=\boldsymbol{Q M P}$ and $\boldsymbol{Y}=r_{\mathrm{M}} \boldsymbol{Q}$, then:

$$
\begin{aligned}
\dot{V}_{1}(t) \leq & \boldsymbol{x}^{T}(t)\left(\boldsymbol{P}(\boldsymbol{A}+\boldsymbol{B})+(\boldsymbol{A}+\boldsymbol{B})^{T} \boldsymbol{P}\right. \\
& \left.+r_{\mathrm{M}}^{2}\left(\boldsymbol{X}^{T}+\boldsymbol{P}\right) \boldsymbol{Y}^{-1}(\boldsymbol{X}+\boldsymbol{P})+\boldsymbol{X}^{T} \boldsymbol{B}+\boldsymbol{B} \boldsymbol{X}\right) \boldsymbol{x}(t) \\
& -2 \boldsymbol{x}^{T}(t) \boldsymbol{X}^{T} \boldsymbol{B} \boldsymbol{x}(t-r(t))+2 \boldsymbol{x}^{T}(t) \boldsymbol{P} \boldsymbol{C} \dot{\boldsymbol{x}}(t-\tau(t)) \\
& +2 \boldsymbol{x}(t) \boldsymbol{P} \boldsymbol{f}(\boldsymbol{x}(t), t)+2 \boldsymbol{x}^{T}(t) \boldsymbol{P} \boldsymbol{g}(\boldsymbol{x}(t-r(t)), t) \\
& +2 \boldsymbol{x}^{T}(t) \boldsymbol{P} \boldsymbol{h}(\dot{\boldsymbol{x}}(t-\tau(t)), t)+\int_{t-r(t)}^{t} \dot{\boldsymbol{x}}^{T}(\xi) \boldsymbol{B}^{T} \boldsymbol{Q B} \dot{\boldsymbol{x}}(\xi) d \xi
\end{aligned}
$$

Noting that using (2), one can easily compute $\dot{V}_{2}(t), \dot{V}_{3}(t)$ and $\dot{V}_{4}(t)$ as:

$$
\begin{aligned}
& \dot{V}_{2}(t)=\dot{\boldsymbol{x}}^{T}(t) \boldsymbol{B}^{T} \boldsymbol{Y B} \dot{\boldsymbol{x}}(t)-\int_{t-r_{\mathrm{M}}}^{t} \dot{\boldsymbol{x}}^{T}(\xi) \boldsymbol{B}^{T} \boldsymbol{Q B} \dot{\boldsymbol{x}}(\xi) d \xi \\
& \dot{V}_{3}(t) \leq \boldsymbol{x}^{T}(t) \boldsymbol{R} \boldsymbol{x}(t)-\left(1-r_{\mathrm{d}}\right) \boldsymbol{x}^{T}(t-r(t)) \boldsymbol{R} \boldsymbol{x}(t-r(t)) \\
& \dot{V}_{4}(t) \leq \dot{\boldsymbol{x}}^{T}(t) \boldsymbol{S} \dot{\boldsymbol{x}}(t)-\left(1-\tau_{\mathrm{d}}\right) \dot{\boldsymbol{x}}^{T}(t-\tau(t)) \boldsymbol{S} \dot{\boldsymbol{x}}(t-\tau(t))
\end{aligned}
$$

Then we have

$$
\begin{aligned}
\dot{V}(t) \leq & \boldsymbol{x}^{T}(t)\left(\boldsymbol{P}(\boldsymbol{A}+\boldsymbol{B})+(\boldsymbol{A}+\boldsymbol{B})^{T} \boldsymbol{P}+\boldsymbol{R}+\boldsymbol{X}^{T} \boldsymbol{B}\right. \\
& \left.+\boldsymbol{B} \boldsymbol{X}+r_{\mathrm{M}}^{2}\left(\boldsymbol{X}^{T}+\boldsymbol{P}\right) \boldsymbol{Y}^{-1}(\boldsymbol{X}+\boldsymbol{P})\right) x(t) \\
& -2 \boldsymbol{x}^{T}(t) \boldsymbol{X}^{T} \boldsymbol{B} \boldsymbol{x}(t-r(t))+2 \boldsymbol{x}^{T}(t) \boldsymbol{P} \boldsymbol{C} \dot{\boldsymbol{x}}(t-\tau(t)) \\
& +2 \boldsymbol{x}^{T}(t) \boldsymbol{P} \boldsymbol{f}\left(\dot{\boldsymbol{x}}((t), t)+2 \boldsymbol{x}^{T} \boldsymbol{P} \boldsymbol{g}(\boldsymbol{x}(t-r(t), t)\right. \\
& +2 \boldsymbol{x}^{T}(t) \boldsymbol{P h}(\dot{\boldsymbol{x}}(t-\tau(t)), t)-\left(1-r_{\mathrm{d}}\right) \boldsymbol{x}^{T}(t-r(t)) \\
& \times \boldsymbol{R} \boldsymbol{x}(t-r(t))-\left(1-\tau_{\mathrm{d}}\right) \dot{\boldsymbol{x}}^{T}(t-\tau(t)) \boldsymbol{S} \dot{\boldsymbol{x}}(t-\tau(t)) \\
& +\dot{\boldsymbol{x}}^{T}(t)\left(\boldsymbol{S}+\boldsymbol{B}^{T} \boldsymbol{Y} \boldsymbol{B}\right) \dot{\boldsymbol{x}}(t)
\end{aligned}
$$

Noting that using (1), we further have:

$$
\dot{V}(t) \leq \boldsymbol{q}^{T}(t) \Xi \boldsymbol{q}(t)
$$

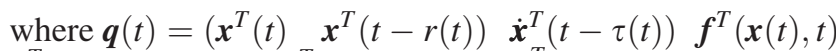
$\left.\boldsymbol{g}^{T}(\boldsymbol{x}(t-r(t)), t) \quad \boldsymbol{h}^{T}(\dot{\boldsymbol{x}}(t-\tau(t)), t)\right)^{T}$ and:

IEE Proc.-Control Theory Appl., Vol. 151, No. 5, September 2004

$$
\Xi=\left(\begin{array}{llllll}
\Xi_{11} & \Xi_{12} & \Xi_{13} & \Xi_{14} & \Xi_{15} & \Xi_{16} \\
\Xi_{12}^{T} & \Xi_{22} & \Xi_{23} & \Xi_{24} & \Xi_{25} & \Xi_{26} \\
\Xi_{13}^{T} & \Xi_{23}^{T} & \Xi_{33} & \Xi_{34} & \Xi_{35} & \Xi_{36} \\
\Xi_{14}^{T} & \Xi_{24}^{T} & \Xi_{34}^{T} & \Xi_{44} & \Xi_{45} & \Xi_{46} \\
\Xi_{15}^{T} & \Xi_{25}^{T} & \Xi_{35}^{T} & \Xi_{45}^{T} & \Xi_{55} & \Xi_{56} \\
\Xi_{16}^{T} & \Xi_{26}^{T} & \Xi_{36}^{T} & \Xi_{46}^{T} & \Xi_{56}^{T} & \Xi_{66}
\end{array}\right)
$$

where

$$
\begin{aligned}
& \boldsymbol{\Xi}_{11}=\boldsymbol{P}(\boldsymbol{A}+\boldsymbol{B})+(\boldsymbol{A}+\boldsymbol{B})^{T} \boldsymbol{P}+\boldsymbol{X}^{T} \boldsymbol{B}+\boldsymbol{B} \boldsymbol{X}+\boldsymbol{R} \\
& +r_{\mathrm{M}}^{2}\left(\boldsymbol{X}^{T}+\boldsymbol{P}\right) \boldsymbol{Y}^{-1}(\boldsymbol{X}+\boldsymbol{P})+\boldsymbol{A}^{T}\left(\boldsymbol{S}+\boldsymbol{B}^{T} \boldsymbol{Y} \boldsymbol{B}\right) \boldsymbol{A} \\
& \boldsymbol{\Xi}_{12}=-\boldsymbol{X}^{T} \boldsymbol{B}+\boldsymbol{A}^{T}\left(\boldsymbol{S}+\boldsymbol{B}^{T} \boldsymbol{Y} \boldsymbol{B}\right) \boldsymbol{B} \\
& \Xi_{13}=\boldsymbol{P C}+\boldsymbol{A}^{T}\left(\boldsymbol{S}+\boldsymbol{B}^{T} \boldsymbol{Y} \boldsymbol{B}\right) \boldsymbol{C} \\
& \Xi_{14}=\boldsymbol{P}+\boldsymbol{A}^{T}\left(\boldsymbol{S}+\boldsymbol{B}^{T} \boldsymbol{Y} \boldsymbol{B}\right) \\
& \boldsymbol{\Xi}_{15}=\boldsymbol{P}+\boldsymbol{A}^{T}\left(\boldsymbol{S}+\boldsymbol{B}^{T} \boldsymbol{Y} \boldsymbol{B}\right) \\
& \boldsymbol{\Xi}_{16}=\boldsymbol{P}+\boldsymbol{A}^{T}\left(\boldsymbol{S}+\boldsymbol{B}^{T} \boldsymbol{Y} \boldsymbol{B}\right) \\
& \boldsymbol{\Xi}_{22}=-\left(1-r_{\mathrm{d}}\right) \boldsymbol{R}+\boldsymbol{B}^{T}\left(\boldsymbol{S}+\boldsymbol{B}^{T} \boldsymbol{Y} \boldsymbol{B}\right) \boldsymbol{B} \\
& \Xi_{23}=\boldsymbol{B}^{T}\left(\boldsymbol{S}+\boldsymbol{B}^{T} \boldsymbol{Y} \boldsymbol{B}\right) \boldsymbol{C} \\
& \Xi_{24}=\boldsymbol{B}^{T}\left(\boldsymbol{S}+\boldsymbol{B}^{T} \boldsymbol{Y} \boldsymbol{B}\right) \\
& \boldsymbol{\Xi}_{25}=\boldsymbol{B}^{T}\left(\boldsymbol{S}+\boldsymbol{B}^{T} \boldsymbol{Y} \boldsymbol{B}\right) \\
& \Xi_{26}=\boldsymbol{B}^{T}\left(\boldsymbol{S}+\boldsymbol{B}^{T} \boldsymbol{Y} \boldsymbol{B}\right) \\
& \Xi_{33}=-\left(1-\tau_{\mathrm{d}}\right) \boldsymbol{S}+\boldsymbol{C}^{T}\left(\boldsymbol{S}+\boldsymbol{B}^{T} \boldsymbol{Y} \boldsymbol{B}\right) \boldsymbol{C} \\
& \Xi_{34}=\boldsymbol{C}^{T}\left(\boldsymbol{S}+\boldsymbol{B}^{T} \boldsymbol{Y} \boldsymbol{B}\right) \\
& \boldsymbol{\Xi}_{35}=\boldsymbol{C}^{T}\left(\boldsymbol{S}+\boldsymbol{B}^{T} \mathbf{Y} \boldsymbol{B}\right) \\
& \Xi_{36}=\boldsymbol{C}^{T}\left(\boldsymbol{S}+\boldsymbol{B}^{T} \boldsymbol{Y B}\right) \\
& \Xi_{44}=\boldsymbol{S}+\frac{1}{1-r_{\mathrm{d}}} \boldsymbol{B}^{T} \boldsymbol{Y} \boldsymbol{B} \\
& \Xi_{45}=\boldsymbol{S}+\boldsymbol{B}^{T} \boldsymbol{Y B} \\
& \Xi_{46}=S+B^{T} Y \boldsymbol{B} \\
& \Xi_{55}=\boldsymbol{S}+\boldsymbol{B}^{T} \boldsymbol{Y B} \\
& \Xi_{56}=\boldsymbol{S}+\boldsymbol{B}^{T} \boldsymbol{Y B} \\
& \Xi_{66}=S+B^{T} Y \boldsymbol{B}
\end{aligned}
$$

By theorem 1.6 in [18], a sufficient condition for asymptotic stability of system (1) is that if $\|\boldsymbol{C}\|+\gamma<1$ and there exist a real matrix $\boldsymbol{X}$, symmetric positive definite matrices $\boldsymbol{P}, \boldsymbol{R}, \boldsymbol{S}$ and $\boldsymbol{Y}$ such that:

$$
\dot{V}(t) \leq \boldsymbol{q}^{T}(t) \Xi \boldsymbol{q}(t)<0
$$

for all $\boldsymbol{q}(t) \neq 0$ satisfying (5), where (7) means that $V(t)$ is negative definite whenever neither $\boldsymbol{x}(t)$ nor $\boldsymbol{x}(t-r(t))$, nor $\dot{\boldsymbol{x}}(t-\tau(t))$ is zero. Using the $S$-procedure [19], we see that this condition is implied by the existence of non-negative scalars $\varepsilon_{1} \geq 0, \varepsilon_{2} \geq 0$ and $\varepsilon_{3} \geq 0$ such that:

$$
\begin{aligned}
\boldsymbol{q}^{T}(t) & \Xi \boldsymbol{q}(t)+\varepsilon_{1}\left(\alpha^{2} \boldsymbol{x}^{T}(t) \boldsymbol{x}(t)-\boldsymbol{f}^{T}(\boldsymbol{x}(t), t) \boldsymbol{f}(\boldsymbol{x}(t), t)\right) \\
& +\varepsilon_{2}\left(\beta^{2} \boldsymbol{x}^{T}(t-r(t)) \boldsymbol{x}(t-r(t))\right. \\
& \left.-\boldsymbol{g}^{T}(\boldsymbol{x}(t-r(t)), t) \boldsymbol{g}(\boldsymbol{x}(t-r(t)), t)\right) \\
& +\varepsilon_{3}\left(\gamma^{2} \dot{\boldsymbol{x}}^{T}(t-\tau(t)) \dot{\boldsymbol{x}}(t-\tau(t))\right. \\
& \left.-\boldsymbol{h}^{T}(\dot{\boldsymbol{x}}(t-\tau(t)), t) \boldsymbol{h}(\dot{\boldsymbol{x}}(t-\tau(t)), t)\right)<0
\end{aligned}
$$

for all $\boldsymbol{q}(t) \neq 0$. Therefore, if $\|\boldsymbol{C}\|+\gamma<1$ and there exist a real matrix $\boldsymbol{X}$, symmetric positive definite matrices $\boldsymbol{P}, \boldsymbol{R}, \boldsymbol{S}$, $\boldsymbol{Y}$ and scalars $\varepsilon_{1} \geq 0, \varepsilon_{2} \geq 0$ and $\varepsilon_{3} \geq 0$ such that the LMI 
(6) is satisfied, then system (1)-(3), with uncertainty (4), is asymptotically stable.

Remark 1: The condition $\|\boldsymbol{C}\|+\gamma<1$ in proposition 1 guarantees that the Lipschitz constant for the right-hand side of (1) with respect to $\dot{\boldsymbol{x}}(t-\tau(t))$ is less than one.

If we choose the following Lyapunov-Krasovskii functional candidate for system (1) as:

$$
\begin{aligned}
V(t)= & \boldsymbol{x}^{T}(t) \boldsymbol{P} \boldsymbol{x}(t)+\int_{t-r(t)}^{t} \boldsymbol{x}^{T}(\xi) \boldsymbol{R} \boldsymbol{x}(\xi) d \xi \\
& +\int_{t-\tau(t)}^{t} \dot{\boldsymbol{x}}^{T}(\xi) \boldsymbol{S} \dot{\boldsymbol{x}}(\xi) d \xi
\end{aligned}
$$

Similar to the proof of proposition 1, we have the following delay-derivative-dependent stability result.

Proposition 2: The system described by (1)-(3), with uncertainty described by (4) is asymptotically stable if $\|\boldsymbol{C}\|+\gamma<1$ and there exist symmetric positive definite matrices $\boldsymbol{P}, \boldsymbol{R}, \boldsymbol{S}$ and scalars $\varepsilon_{1} \geq 0, \varepsilon_{2} \geq 0$ and $\varepsilon_{3} \geq 0$ such that the following LMI holds:

$$
\left(\begin{array}{ccccccc}
(1,1) & \boldsymbol{P B} & \boldsymbol{P} \boldsymbol{C} & \boldsymbol{P} & \boldsymbol{P} & \boldsymbol{P} & \boldsymbol{A}^{T} \boldsymbol{S} \\
\boldsymbol{B}^{T} \boldsymbol{P} & (2,2) & 0 & 0 & 0 & 0 & \boldsymbol{B}^{T} \boldsymbol{S} \\
\boldsymbol{C}^{T} \boldsymbol{P} & 0 & (3,3) & 0 & 0 & 0 & \boldsymbol{C}^{T} \boldsymbol{S} \\
\boldsymbol{P} & 0 & 0 & -\varepsilon_{1} \boldsymbol{I} & 0 & 0 & \boldsymbol{S} \\
\boldsymbol{P} & 0 & 0 & 0 & -\varepsilon_{2} \boldsymbol{I} & 0 & \boldsymbol{S} \\
\boldsymbol{P} & 0 & 0 & 0 & 0 & -\varepsilon_{3} \boldsymbol{I} & \boldsymbol{S} \\
\boldsymbol{S A} & \boldsymbol{S B} & \boldsymbol{S C} & \boldsymbol{S} & \boldsymbol{S} & \boldsymbol{S} & -\boldsymbol{S}
\end{array}\right)<0
$$

where

$$
\begin{aligned}
& (1,1) \triangleq \boldsymbol{A}^{T} \boldsymbol{P}+\boldsymbol{P A}+\boldsymbol{R}+\varepsilon_{1} \alpha^{2} \boldsymbol{I} \\
& (2,2) \triangleq-\left(1-r_{\mathrm{d}}\right) \boldsymbol{R}+\varepsilon_{2} \beta^{2} \boldsymbol{I} \\
& (3,3) \triangleq-\left(1-\tau_{\mathrm{d}}\right) \boldsymbol{S}+\varepsilon_{3} \gamma^{2} \boldsymbol{I}
\end{aligned}
$$

If $\boldsymbol{C} \equiv 0$ and $\boldsymbol{h}(\dot{\boldsymbol{x}}(t-\tau(t)), t) \equiv 0$, then system (1) reduces to the following system:

$\dot{\boldsymbol{x}}(t)=\boldsymbol{A} \boldsymbol{x}(t)+\boldsymbol{B} \boldsymbol{x}(t-r(t))+\boldsymbol{f}(\boldsymbol{x}(t), t)+\boldsymbol{g}(\boldsymbol{x}(t-r(t)), t)$

with initial condition

$$
\boldsymbol{x}\left(t_{0}+\theta\right)=\varphi(\theta) \quad \forall \theta \in\left[-r_{\mathrm{M}}, 0\right]
$$

According to proposition 1, we have the following corollary for the delay-dependent stability of system (9) and (10).

Corollary 1: The system described by (9), (10) and (3), with uncertainty described by $(4 a)$ and $(4 b)$ is asymptotically stable if there exists a real matrix $\boldsymbol{X}$, symmetric positive definite matrices $\boldsymbol{P}, \boldsymbol{R}, \boldsymbol{Y}$ and scalars $\varepsilon_{1} \geq 0$, $\varepsilon_{2} \geq 0$ such that the following LMI holds:

$$
\left(\begin{array}{cccccc}
(1,1) & -\boldsymbol{X}^{T} \boldsymbol{B} & \boldsymbol{P} & \boldsymbol{P} & \boldsymbol{A}^{T} \boldsymbol{B}^{T} \boldsymbol{Y} & r_{\mathrm{M}}\left(\boldsymbol{X}^{T}+\boldsymbol{P}\right) \\
-\boldsymbol{B}^{T} \boldsymbol{X} & (2,2) & 0 & 0 & \boldsymbol{B}^{T} \boldsymbol{B}^{T} \boldsymbol{Y} & 0 \\
\boldsymbol{P} & 0 & -\varepsilon_{1} \boldsymbol{I} & 0 & \boldsymbol{B}^{T} \boldsymbol{Y} & 0 \\
\boldsymbol{P} & 0 & 0 & -\varepsilon_{2} \boldsymbol{I} & \boldsymbol{B}^{T} \boldsymbol{Y} & 0 \\
\boldsymbol{Y} \boldsymbol{B} \boldsymbol{A} & \boldsymbol{Y} \boldsymbol{B} \boldsymbol{B} & \boldsymbol{Y} \boldsymbol{B} & \boldsymbol{Y} \boldsymbol{B} & -\boldsymbol{Y} & 0 \\
r_{\mathrm{M}}(\boldsymbol{X}+\boldsymbol{P}) & 0 & 0 & 0 & 0 & -\boldsymbol{Y}
\end{array}\right)<0
$$

where

$$
\begin{aligned}
& (1,1) \triangleq(\boldsymbol{A}+\boldsymbol{B})^{T} \boldsymbol{P}+\boldsymbol{P}(\boldsymbol{A}+\boldsymbol{B})+\boldsymbol{R}+\boldsymbol{X}^{T} \boldsymbol{B}+\boldsymbol{B}^{T} \boldsymbol{X}+\varepsilon_{1} \alpha^{2} \boldsymbol{I} \\
& (2,2) \triangleq-\left(1-r_{\mathrm{d}}\right) \boldsymbol{R}+\varepsilon_{2} \beta^{2} \boldsymbol{I}
\end{aligned}
$$

Remark 2: If $\boldsymbol{f}(\boldsymbol{x}(t), t) \equiv 0, \boldsymbol{g}(\boldsymbol{x}(t-h(t)), t) \equiv 0$, and $r(t)$ $\equiv r$ (constant), system (9) and (10) becomes:

$$
\dot{\boldsymbol{x}}(t)=\boldsymbol{A} \boldsymbol{x}(t)+\boldsymbol{B} \boldsymbol{x}(t-r)
$$

with initial condition

$$
\boldsymbol{x}\left(t_{0}+\theta\right)=\varphi(\theta) \quad \forall \theta \in[-r, 0]
$$

By corollary 1, we can conclude that system (12) and (13) is asymptotically stable if there exists a real matrix $\boldsymbol{X}$, and symmetric positive definite matrices $\boldsymbol{P}, \boldsymbol{R}$, and $\boldsymbol{Y}$ such that:

$$
\left(\begin{array}{cccc}
(1,1) & -\boldsymbol{X}^{T} \boldsymbol{B} & \boldsymbol{A}^{T} \boldsymbol{B}^{T} \boldsymbol{Y} & r\left(\boldsymbol{X}^{T}+\boldsymbol{P}\right) \\
-\boldsymbol{B}^{T} \boldsymbol{X} & -\boldsymbol{R} & \boldsymbol{B}^{T} \boldsymbol{B}^{T} \boldsymbol{Y} & 0 \\
\boldsymbol{Y} \boldsymbol{B} \boldsymbol{A} & \boldsymbol{Y B} \boldsymbol{B} & -\boldsymbol{Y} & 0 \\
r(\boldsymbol{X}+\boldsymbol{P}) & 0 & 0 & -\boldsymbol{Y}
\end{array}\right)<0
$$

where

$$
(1,1) \triangleq(\boldsymbol{A}+\boldsymbol{B})^{T} \boldsymbol{P}+\boldsymbol{P}(\boldsymbol{A}+\boldsymbol{B})+\boldsymbol{R}+\boldsymbol{X}^{T} \boldsymbol{B}+\boldsymbol{B}^{T} \boldsymbol{X}
$$

Then theorem 1 in [16] is recovered.

By proposition 2, the following corollary is easily obtained for the delay-derivative-dependent stability of system (9) and (10).

Corollary 2: The system described by (9), (10) and (3), with uncertainty described by $(4 a)$ and $(4 b)$ is asymptotically stable if there exist symmetric positive definite matrices $\boldsymbol{P}$, $\boldsymbol{R}$, and scalars $\varepsilon_{1} \geq 0, \varepsilon_{2} \geq 0$ such that the following LMI holds:

$$
\left(\begin{array}{cccc}
\boldsymbol{A}^{T} \boldsymbol{P}+\boldsymbol{P} \boldsymbol{A}+\boldsymbol{R}+\varepsilon_{1} \alpha^{2} \boldsymbol{I} & \boldsymbol{P B} & \boldsymbol{P} & \boldsymbol{P} \\
\boldsymbol{B}^{T} \boldsymbol{P} & -\left(1-r_{\mathrm{d}}\right) \boldsymbol{R}+\varepsilon_{2} \beta^{2} \boldsymbol{I} & 0 & 0 \\
\boldsymbol{P} & 0 & -\varepsilon_{1} \boldsymbol{I} & 0 \\
\boldsymbol{P} & 0 & 0 & -\varepsilon_{2} \boldsymbol{I}
\end{array}\right)<0
$$

Remark 3: Corollary 2 is theorem 1 in [13]. This means that proposition 2 extends the result in [13] to neutral systems.

\section{Norm-bounded uncertainty}

In this Section we will handle the case that $\boldsymbol{f}(\boldsymbol{x}(t), t)$, $\boldsymbol{g}(\boldsymbol{x}(t-h(t)), t)$ and $\boldsymbol{h}(\dot{\boldsymbol{x}}(t-\tau(t)), t)$ are norm-bounded uncertainties that are well known in robust control of uncertain systems [17]. Then system (1) becomes the following system:

$$
\begin{aligned}
\dot{\boldsymbol{x}}(t)= & \left(\boldsymbol{A}+\boldsymbol{L} \boldsymbol{F}(t) \boldsymbol{E}_{\mathrm{a}}\right) \boldsymbol{x}(t)+\left(\boldsymbol{B}+\boldsymbol{L F}(t) \boldsymbol{E}_{\mathrm{b}}\right) \boldsymbol{x}(t-r(t)) \\
& +\left(\boldsymbol{C}+\boldsymbol{L} \boldsymbol{F}(t) \boldsymbol{E}_{\mathrm{c}}\right) \dot{\boldsymbol{x}}(t-\tau(t))
\end{aligned}
$$

where $\boldsymbol{F}(t) \in \mathbb{R}^{p \times q}$ is an unknown real and possibly time-varying matrix with Lebesgue measurable elements satisfying:

$$
\boldsymbol{F}^{T}(t) \boldsymbol{F}(t) \leq \boldsymbol{I}
$$

and $\boldsymbol{L}, \boldsymbol{E}_{\mathrm{a}}, \boldsymbol{E}_{\mathrm{b}}$ and $\boldsymbol{E}_{\mathrm{c}}$ are known real constant matrices which characterise how the uncertainty enters the nominal matrices $\boldsymbol{A}, \boldsymbol{B}$ and $\boldsymbol{C}$. 
System (16) can be written as:

$$
\begin{gathered}
\dot{\boldsymbol{x}}(t)=\boldsymbol{A} \boldsymbol{x}(t)+\boldsymbol{B} \boldsymbol{x}(t-r(t))+\boldsymbol{C} \dot{\boldsymbol{x}}(t-\tau(t))+\boldsymbol{L} \boldsymbol{u} \\
\boldsymbol{y}=\boldsymbol{E}_{\mathrm{a}} \boldsymbol{x}(t)+\boldsymbol{E}_{\mathrm{b}} \boldsymbol{x}(t-r(t))+\boldsymbol{E}_{\mathrm{c}} \dot{\boldsymbol{x}}(t-\tau(t))
\end{gathered}
$$

with the constraint

$$
\boldsymbol{u}=\boldsymbol{F}(t) \boldsymbol{y}
$$

We further rewrite (18) and (19) as:

$$
\begin{aligned}
\dot{\boldsymbol{x}}(t) & -\boldsymbol{A} \boldsymbol{x}(t)+\boldsymbol{B} \boldsymbol{x}(t-r(t))+\boldsymbol{C} \dot{\boldsymbol{x}}(t-\tau(t))+\boldsymbol{L} \boldsymbol{u} \\
\boldsymbol{u}^{T} \boldsymbol{u} \leq & \left(\boldsymbol{E}_{\mathrm{a}} \boldsymbol{x}(t)+\boldsymbol{E}_{\mathrm{b}} \boldsymbol{x}(t-r(t))+\boldsymbol{E}_{\mathrm{c}} \dot{\boldsymbol{x}}(t-\tau(t))\right)^{T}\left(\boldsymbol{E}_{\mathrm{a}} \boldsymbol{x}(t)\right. \\
& \left.+\boldsymbol{E}_{\mathrm{b}} \boldsymbol{x}(t-r(t))+\boldsymbol{E}_{\mathrm{c}} \dot{\boldsymbol{x}}(t-\tau(t))\right)
\end{aligned}
$$

We now state and establish the following delaydependent stability result.

Proposition 3: The system described by (16), (17), (2) and (3) is asymptotically stable if there exist a scalar $\delta>0$ satisfying $\delta \boldsymbol{I}-\boldsymbol{L}^{T} \boldsymbol{L}>0$, and a real matrix $\tilde{\boldsymbol{X}}$, symmetric positive definite matrices $\tilde{\boldsymbol{P}}, \tilde{\boldsymbol{R}}, \tilde{\boldsymbol{S}}$ and $\tilde{\boldsymbol{Y}}$ such that the following LMIs hold:

$$
\begin{aligned}
& \left(\begin{array}{cc}
\boldsymbol{C}^{T} \boldsymbol{C}-\boldsymbol{I}+\delta \boldsymbol{E}_{\mathrm{c}}^{T} \boldsymbol{E}_{\mathrm{c}} & \boldsymbol{C}^{T} \boldsymbol{L} \\
\boldsymbol{L} \boldsymbol{C} & -\left(\delta \boldsymbol{I}-\boldsymbol{L}^{T} \boldsymbol{L}\right)
\end{array}\right)<0
\end{aligned}
$$

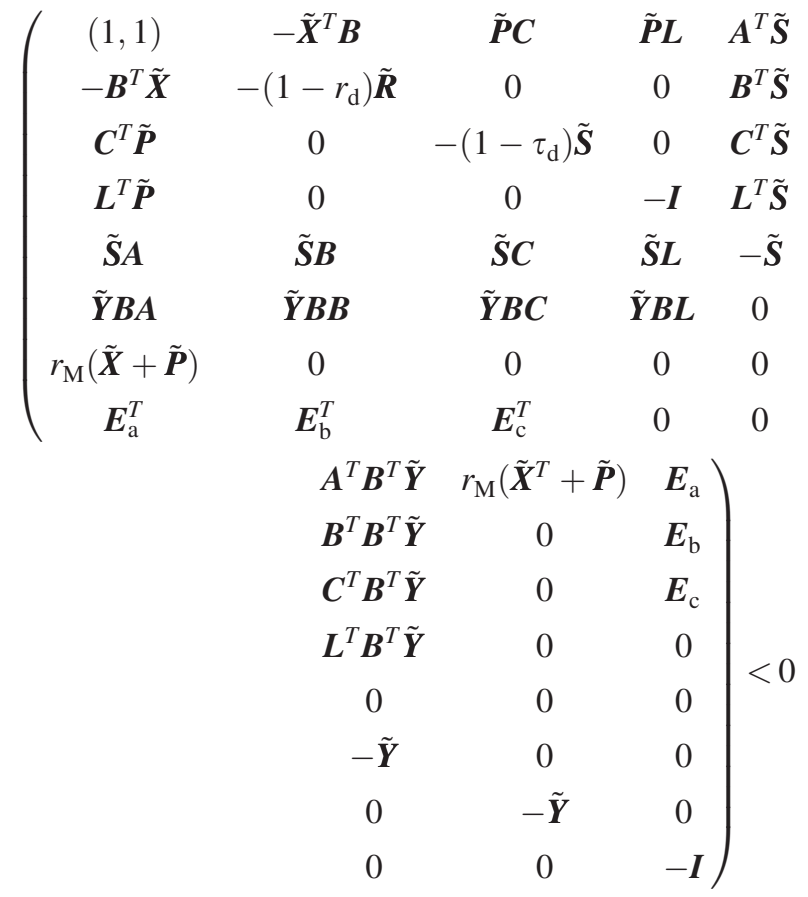

where

$$
(1,1) \triangleq(\boldsymbol{A}+\boldsymbol{B})^{T} \tilde{\boldsymbol{P}}+\tilde{\boldsymbol{P}}(\boldsymbol{A}+\boldsymbol{B})+\tilde{\boldsymbol{R}}+\tilde{\boldsymbol{X}}^{T} \boldsymbol{B}+\boldsymbol{B}^{T} \tilde{\boldsymbol{X}}
$$

Remark 4: Although norm-bounded uncertainties can be treated as a special case of nonlinear parameter perturbations, one can get a less conservative result using proposition 3 than proposition 1 .

Similar to proposition $2.2(b)$ in [20], we have the following lemma.

Lemma 2: Let $\boldsymbol{A}, \boldsymbol{L}, \boldsymbol{E}$ and $\boldsymbol{F}(t)$ be real matrices of appropriate dimensions with $\boldsymbol{F}^{T}(t) \boldsymbol{F}(t) \leq \boldsymbol{I}$. Then for any symmetric positive definite matrix $\boldsymbol{P}>0$ and a scalar $\varepsilon>0$ such that $\varepsilon \boldsymbol{I}-\boldsymbol{L}^{T} \boldsymbol{P} \boldsymbol{L}>0$, the following inequality holds:

$$
\begin{aligned}
(\boldsymbol{A} & +\boldsymbol{L} \boldsymbol{F}(t) \boldsymbol{E})^{T} \boldsymbol{P}(\boldsymbol{A}+\boldsymbol{L F}(t) \boldsymbol{E}) \\
& \leq \boldsymbol{A}^{T} \boldsymbol{P} \boldsymbol{A}+\boldsymbol{A}^{T} \boldsymbol{P} \boldsymbol{L}\left(\varepsilon \boldsymbol{I}-\boldsymbol{L}^{T} \boldsymbol{P} \boldsymbol{L}\right)^{-1} \boldsymbol{L}^{T} \boldsymbol{P} \boldsymbol{A}+\varepsilon \boldsymbol{E}^{T} \boldsymbol{E}
\end{aligned}
$$

Proof of Proposition 3: Similar to the proof of proposition 1, we can conclude that the system described by (16), (17), (2) and (3) is asymptotically stable if $\left(\boldsymbol{C}+\boldsymbol{L F}(t) \boldsymbol{E}_{\mathrm{c}}\right)^{T}(\boldsymbol{C}+$ $\left.\boldsymbol{L} \boldsymbol{F}(t) \boldsymbol{E}_{\mathrm{c}}\right)<\boldsymbol{I}$ (which means that $\left\|\boldsymbol{C}+\boldsymbol{L} \boldsymbol{F}(t) \boldsymbol{E}_{\mathrm{c}}\right\|<1$ ) and there exist a real matrix $\boldsymbol{X}$, symmetric positive definite matrices $\boldsymbol{P}, \boldsymbol{R}, \boldsymbol{S}, \boldsymbol{Y}$ and a scalar $\varepsilon \geq 0$ such that the following LMI holds:

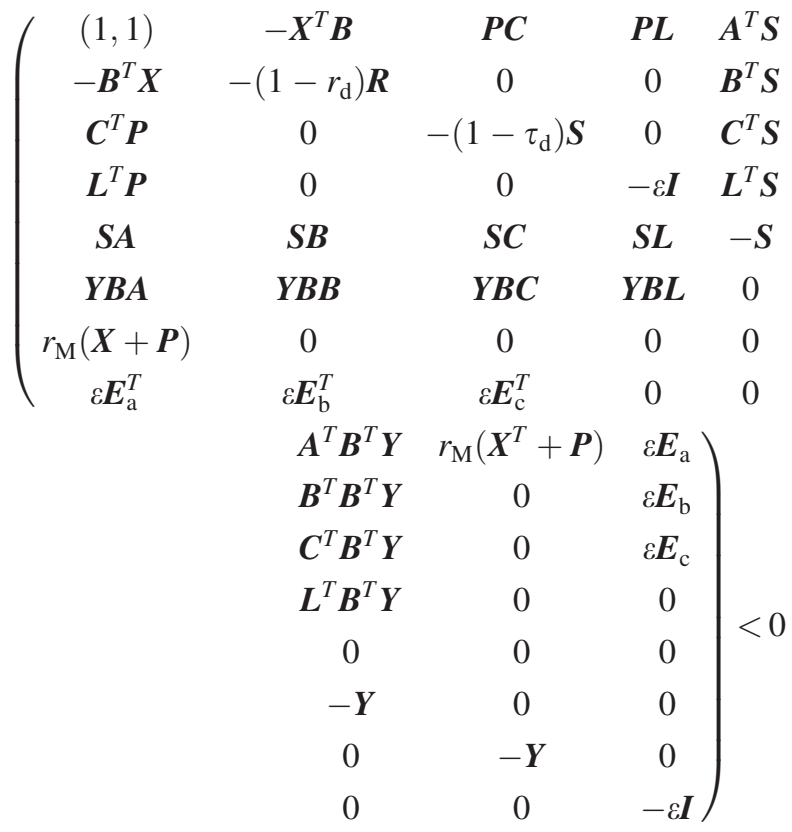

where

$$
(1,1) \triangleq(\boldsymbol{A}+\boldsymbol{B})^{T} \boldsymbol{P}+\boldsymbol{P}(\boldsymbol{A}+\boldsymbol{B})+\boldsymbol{R}+\boldsymbol{X}^{T} \boldsymbol{B}+\boldsymbol{B}^{T} \boldsymbol{X}
$$

Noting that (23) implies that $\varepsilon>0$ and introducing new variables $\tilde{\boldsymbol{P}}=\varepsilon^{-1} \boldsymbol{P}, \tilde{\boldsymbol{R}}=\varepsilon^{-1} \boldsymbol{R}, \tilde{\boldsymbol{S}}=\varepsilon^{-1} \boldsymbol{S}, \tilde{\boldsymbol{Y}}=\varepsilon^{-1} \boldsymbol{Y}, \tilde{\boldsymbol{X}}=$ $\varepsilon^{-1} \boldsymbol{X}$ yields (22).

By lemma 2, we have:

$$
\begin{aligned}
(\boldsymbol{C} & \left.+\boldsymbol{L} \boldsymbol{F}(t) \boldsymbol{E}_{\mathrm{c}}\right)^{T}\left(\boldsymbol{C}+\boldsymbol{L} \boldsymbol{F}(t) \boldsymbol{E}_{\mathrm{c}}\right) \\
& \leq \boldsymbol{C}^{T} \boldsymbol{C}+\boldsymbol{C}^{T} \boldsymbol{L}\left(\varepsilon \boldsymbol{I}-\boldsymbol{L}^{T} \boldsymbol{L}\right)^{-1} \boldsymbol{L}^{T} \boldsymbol{C}+\varepsilon \boldsymbol{E}_{\mathrm{c}}^{T} \boldsymbol{E}_{\mathrm{c}}
\end{aligned}
$$

If (21) is satisfied, then we have that $\left(\boldsymbol{C}+\boldsymbol{L F}(t) \boldsymbol{E}_{\mathrm{c}}\right)^{T}(\boldsymbol{C}+$ $\left.\boldsymbol{L F}(t) \boldsymbol{E}_{\mathrm{c}}\right)<\boldsymbol{I}$

Similar to proposition 2 , we have the following delayderivative-dependent stability result.

Proposition 4: The system described by (16), (17), (2) and (3) is asymptotically stable if there exist a scalar $\delta>0$ satisfying $\delta \boldsymbol{I}-\boldsymbol{L}^{T} \boldsymbol{L}>0$, and symmetric positive definite matrices $\tilde{\boldsymbol{P}}, \tilde{\boldsymbol{R}}, \tilde{\boldsymbol{S}}$, such that (21) and the following LMI is satisfied:

$$
\left(\begin{array}{cccccc}
(1,1) & \tilde{\boldsymbol{P}} \boldsymbol{B} & \tilde{\boldsymbol{P}} \boldsymbol{C} & \tilde{\boldsymbol{P}} \boldsymbol{L} & \boldsymbol{A}^{T} \tilde{\boldsymbol{S}} & \boldsymbol{E}_{\mathrm{a}} \\
\boldsymbol{B}^{T} \tilde{\boldsymbol{P}} & (2,2) & 0 & 0 & \boldsymbol{B}^{T} \tilde{\boldsymbol{S}} & \boldsymbol{E}_{\mathrm{b}} \\
\boldsymbol{C}^{T} \tilde{\boldsymbol{P}} & 0 & (3,3) & 0 & \boldsymbol{C}^{T} \tilde{\boldsymbol{S}} & \boldsymbol{E}_{\mathrm{c}} \\
\boldsymbol{L}^{T} \tilde{\boldsymbol{P}} & 0 & 0 & -\boldsymbol{I} & \boldsymbol{L}^{T} \tilde{\boldsymbol{S}} & 0 \\
\tilde{\boldsymbol{S}} \boldsymbol{A} & \tilde{\boldsymbol{S}} \boldsymbol{B} & \tilde{\boldsymbol{S}} \boldsymbol{C} & \tilde{\boldsymbol{S}} \boldsymbol{L} & -\tilde{\boldsymbol{S}} & 0 \\
\boldsymbol{E}_{\mathrm{a}}^{T} & \boldsymbol{E}_{\mathrm{b}}^{T} & \boldsymbol{E}_{\mathrm{c}}^{T} & 0 & 0 & -\boldsymbol{I}
\end{array}\right)<0
$$


where

$$
\begin{aligned}
& (1,1) \triangleq A^{T} \tilde{\boldsymbol{P}}+\tilde{\boldsymbol{P}} \boldsymbol{A}+\tilde{\boldsymbol{R}} \\
& (2,2) \triangleq-\left(1-r_{\mathrm{d}}\right) \tilde{\boldsymbol{R}} \\
& (3,3) \triangleq-\left(1-\tau_{\mathrm{d}}\right) \tilde{\boldsymbol{S}}
\end{aligned}
$$

If $\boldsymbol{C} \equiv 0$ and $\boldsymbol{E}_{\mathrm{c}} \equiv 0$, then system (16) reduces to the following system:

$$
\dot{\boldsymbol{x}}(t)=\left(\boldsymbol{A}+\boldsymbol{L F}(t) \boldsymbol{E}_{\mathrm{a}}\right) \boldsymbol{x}(t)+\left(\boldsymbol{B}+\boldsymbol{L F}(t) \boldsymbol{E}_{\mathrm{b}}\right) \boldsymbol{x}(t-r(t))
$$

with initial condition

$$
\boldsymbol{x}\left(t_{0}+\theta\right)=\boldsymbol{\varphi}(\theta) \quad \forall \theta \in\left[-r_{\mathrm{M}}, 0\right]
$$

For the stability of system (25) and (26), in light of propositions 3 and 4, we have the following corollaries.

Corollary 3: (Delay-dependent stability). The system described by (25), (26), (17) and (2) has an asymptotically stable dependence if there exist a real matrix $\tilde{\boldsymbol{X}}$, symmetric positive definite matrices $\tilde{\boldsymbol{P}}, \tilde{\boldsymbol{R}}$ and $\tilde{\boldsymbol{Y}}$ such that the following LMI holds:

$$
\begin{aligned}
& \left(\begin{array}{cccc}
(1,1) & -\tilde{\boldsymbol{X}}^{T} \boldsymbol{B} & \tilde{\boldsymbol{P}} \boldsymbol{L} & \boldsymbol{A}^{T} \boldsymbol{B}^{T} \tilde{\boldsymbol{Y}} \\
-\boldsymbol{B}^{T} \tilde{\boldsymbol{X}} & -\left(1-r_{\mathrm{d}}\right) \tilde{\boldsymbol{R}} & 0 & \boldsymbol{B}^{T} \boldsymbol{B}^{T} \tilde{\boldsymbol{Y}} \\
\boldsymbol{L}^{T} \tilde{\boldsymbol{P}} & 0 & -\boldsymbol{I} & \boldsymbol{L}^{T} \boldsymbol{B}^{T} \tilde{\boldsymbol{Y}} \\
\tilde{\boldsymbol{Y}} \boldsymbol{B} \boldsymbol{A} & \tilde{\boldsymbol{Y}} \boldsymbol{B} \boldsymbol{B} & \tilde{\boldsymbol{Y}} \boldsymbol{B} \boldsymbol{L} & -\tilde{\boldsymbol{Y}} \\
r_{\mathrm{M}}(\tilde{\boldsymbol{X}}+\tilde{\boldsymbol{P}}) & 0 & 0 & 0 \\
\boldsymbol{E}_{a}^{T} & \boldsymbol{E}_{\mathrm{b}}^{T} & 0 & 0
\end{array}\right. \\
& \left.\begin{array}{cc}
r_{\mathrm{M}}\left(\tilde{\boldsymbol{X}}^{T}+\tilde{\boldsymbol{P}}\right) & \boldsymbol{E}_{\mathrm{a}} \\
0 & \boldsymbol{E}_{\mathrm{b}} \\
0 & 0 \\
0 & 0 \\
-\tilde{\boldsymbol{Y}} & 0 \\
0 & -\boldsymbol{I}
\end{array}\right)<0
\end{aligned}
$$

where

$$
(1,1) \triangleq(\boldsymbol{A}+\boldsymbol{B})^{T} \boldsymbol{P}+\boldsymbol{P}(\boldsymbol{A}+\boldsymbol{B})+\boldsymbol{R}+\boldsymbol{X}^{T} \boldsymbol{B}+\boldsymbol{B}^{T} \boldsymbol{X}
$$

Corollary 4: (Delay-derivative-dependent stability). The system described by (25), (26), (17) and (2) is asymptotically stable if there exist symmetric positive definite matrices $\tilde{\boldsymbol{P}}$ and $\tilde{\boldsymbol{R}}$ such that the following LMI is satisfied:

$$
\left(\begin{array}{cccc}
\boldsymbol{A}^{T} \tilde{\boldsymbol{P}}+\tilde{\boldsymbol{P}} \boldsymbol{A}+\tilde{\boldsymbol{R}} & \tilde{\boldsymbol{P}} \boldsymbol{B} & \tilde{\boldsymbol{P}} \boldsymbol{L} & \boldsymbol{E}_{\mathrm{a}} \\
\boldsymbol{B}^{T} \tilde{\boldsymbol{P}} & -\left(1-r_{\mathrm{d}}\right) \tilde{\boldsymbol{R}} & 0 & \boldsymbol{E}_{\mathrm{b}} \\
\boldsymbol{L}^{T} \tilde{\boldsymbol{P}} & 0 & -\boldsymbol{I} & 0 \\
\boldsymbol{E}_{\mathrm{a}}^{T} & \boldsymbol{E}_{\mathrm{b}}^{T} & 0 & -\boldsymbol{I}
\end{array}\right)<0
$$

Remark 5: It should be pointed out that the results in propositions 1 and 3 can be further improved by instead of using Park's inequality, one uses the inequality (lemma 1) in [21] to estimate the bound of the cross-term $-2 \boldsymbol{x}^{T}(t) \boldsymbol{P B} \int_{t-r(t)}^{t} \dot{\boldsymbol{x}}(\xi) d \xi$.

\section{Examples}

In order to use proposition 1 to test the stability of the system described by (1)-(3), with nonlinear parameter perturbations described by (4), a Matlab $m$-function is written which automatically generates LMI (6), and then solves this LMI using the LMI solver FEASP in the Matlab

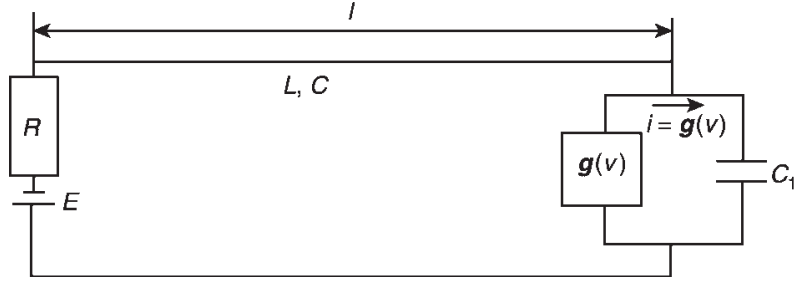

Fig. 1 System with a lossless transmission line

LMI toolbox [22]. The inputs to the function are system matrices $\boldsymbol{A}, \boldsymbol{B}, \boldsymbol{C}$, and $r_{\mathrm{d}}, r_{\mathrm{M}}, \tau_{\mathrm{d}}$, and $\alpha, \beta, \gamma$. The function returns whether or not the LMI is feasible. If feasible, it also gives symmetric positive definite matrices $\boldsymbol{P}, \boldsymbol{R}, \boldsymbol{S}, \boldsymbol{Y}$ and scalars $\varepsilon_{1} \geq 0, \varepsilon_{2} \geq 0$ and $\varepsilon_{3} \geq 0$ as outputs. We also have written Matlab $m$-functions for the remaining propositions and corollaries. The following examples are generated using those MATLAB $m$-functions.

Example 1: Let the system consist of a long electrical cable of length $l$, one end of which is connected to a power source $E$ with resistance $R$, while the other end is connected to an oscillating circuit formed by a condenser $C_{1}$ and a nonlinear element, the volt-ampere characteristic of which is $i=\boldsymbol{g}(v)$, see Fig. 1. Let $L$ and $C$ denote the linear inductance and capacitance of the cable, respectively, and assume that it is lossless. The processes in such a system are described by the following system of partial differential equations:

$$
\begin{gathered}
L \frac{\partial i(x, t)}{\partial t}=-\frac{\partial v(x, t)}{\partial x} C \frac{\partial v(x, t)}{\partial t}=-\frac{\partial i(x, t)}{\partial x} \\
0<x<l, t>0
\end{gathered}
$$

with boundary conditions

$$
E-v(0, t)-R i(0, t)=0 \quad C_{1} \frac{d v(l, t)}{d t}=i(l, t)-\boldsymbol{g}(v(l, t))
$$

Let $s=1 / \sqrt{L C}, \quad z=\sqrt{L / C}, \quad K=(z-R) /(z+R)$ and $\alpha=2 E /(z+R)$. Using transformations yields [3]:

$$
\dot{\boldsymbol{u}}(t)-K \dot{\boldsymbol{u}}(t-2 l / s)=\boldsymbol{f}(\boldsymbol{u}(t), \boldsymbol{u}(t-2 l / s))
$$

where

$$
\begin{aligned}
C_{1} \boldsymbol{f}(\boldsymbol{u}(t), \boldsymbol{u}(t-r))= & \alpha-\frac{1}{z} \boldsymbol{u}(t)-\frac{K}{z} \boldsymbol{u}(t-r) \\
& -\boldsymbol{g}(u(t))+K \boldsymbol{g}(u(t-r))
\end{aligned}
$$

Letting $\boldsymbol{y}(t)=\boldsymbol{u}(t)-(\alpha z) /(1+K)$, we further have:

$$
\begin{aligned}
\dot{\boldsymbol{y}}(t)-K \dot{\boldsymbol{y}}(t-2 l / s)= & -\boldsymbol{y}(t) /\left(C_{1} z\right)-K \boldsymbol{y}(t-2 l / s) /\left(C_{1} z\right) \\
& -\boldsymbol{g}(\boldsymbol{y}(t))+K \boldsymbol{g}(\boldsymbol{y}(t-2 l / s))
\end{aligned}
$$

Choosing $l=0.1006, L=0.2, C=0.1, R=0.12$, and $C_{1}=0.1$, and assuming that $\|\boldsymbol{g}(\boldsymbol{y}(t))\| \leq 0.1\|\boldsymbol{y}(t)\|$, by proposition 1 we can conclude that the considered system is asymptotically stable.

Example 2: Consider system (1) with:

$$
\begin{aligned}
& \boldsymbol{A}=\left(\begin{array}{cc}
-1.2 & 0.1 \\
-0.1 & -1
\end{array}\right) \boldsymbol{B}=\left(\begin{array}{cc}
-0.6 & 0.7 \\
-1 & -0.8
\end{array}\right) \boldsymbol{C}=\left(\begin{array}{ll}
c & 0 \\
0 & c
\end{array}\right) \\
& \|\boldsymbol{f}(\boldsymbol{x}(t), t)\| \leq \alpha\|\boldsymbol{x}(t)\| \\
& \|\boldsymbol{g}(\boldsymbol{x}(t-r(t)), t)\| \leq \beta\|\boldsymbol{x}(t-r(t))\| \text { and } \\
& \|\boldsymbol{h}(\dot{\boldsymbol{x}}(t-\tau(t)), t)\| \leq \gamma\|\dot{\boldsymbol{x}}(t-\tau(t))\|
\end{aligned}
$$

where $0 \leq|c|<1, \alpha \geq 0, \beta \geq 0, \gamma \geq 0$. 
Table 1: Bound $r_{M}$ for $c \equiv 0$ and $h(\dot{x}(t-\tau(t)), t) \equiv 0$

\begin{tabular}{lcccc}
\hline & $\alpha=0, \beta=0.1$ & \multicolumn{2}{c}{$\alpha=0.1, \beta=0.1$} \\
& $r_{\mathrm{d}}=0$ & $r_{\mathrm{d}}=0.5$ & $r_{\mathrm{d}}=0$ & $r_{\mathrm{d}}=0.5$ \\
\hline Cao and Lam [13] & 0.6811 & 0.5467 & 0.6129 & 0.4950 \\
Han [15] & 1.3279 & 0.6743 & 1.2503 & 0.5716 \\
This work & 2.7424 & 1.1365 & 1.8753 & 0.9952 \\
\hline
\end{tabular}

Table 2: Bound $r_{\mathrm{M}}$ for $h(\dot{x}(t-\tau(t)), t) \equiv 0, \tau_{\mathrm{d}}=0$ and different $c$

\begin{tabular}{lllll}
\hline & \multicolumn{2}{c}{$\alpha=0, \beta=0.1$} & \multicolumn{2}{c}{$\alpha=0.1, \beta=0.1$} \\
& $r_{\mathrm{d}}=0$ & $r_{\mathrm{d}}=0.5$ & $r_{\mathrm{d}}=0$ & $r_{\mathrm{d}}=0.5$ \\
\hline$|c|=0.1$ & 2.0366 & 0.9328 & 1.4753 & 0.8148 \\
$|c|=0.2$ & 1.5009 & 0.7429 & 1.1390 & 0.6460 \\
$|c|=0.3$ & 1.0924 & 0.5680 & 0.8587 & 0.4897 \\
$|c|=0.4$ & 0.7774 & 0.4090 & 0.6260 & 0.3470 \\
$|c|=0.5$ & 0.5314 & 0.2670 & 0.4312 & 0.2188 \\
$|c|=0.6$ & 0.3342 & 0.1430 & 0.2673 & 0.1059 \\
$|c|=0.7$ & 0.1765 & 0.0374 & 0.1336 & 0.0090 \\
$|c|=0.8$ & 0.0566 & - & 0.0302 & -
\end{tabular}

Table 3: Bound $r_{M}$ for $c=0.1$ and $\tau_{d}=0$

\begin{tabular}{lllll}
\hline & \multicolumn{2}{c}{$\alpha=0, \beta=0.1$} & \multicolumn{2}{c}{$\alpha=0.1, \beta=0.1$} \\
& $r_{\mathrm{d}}=0$ & $r_{\mathrm{d}}=0.5$ & $r_{\mathrm{d}}=0$ & $r_{\mathrm{d}}=0.5$ \\
\hline$\gamma=0.0$ & 2.0366 & 0.9328 & 1.4753 & 0.8148 \\
$\gamma=0.1$ & 1.4937 & 0.7402 & 1.1356 & 0.6439 \\
$\gamma=0.2$ & 1.0838 & 0.5637 & 0.8451 & 0.4864 \\
$\gamma=0.3$ & 0.7697 & 0.4042 & 0.6215 & 0.3433 \\
\hline
\end{tabular}

Table 4: Bound $r_{M}$ for $c=0.1$ and $\tau_{d}=0.5$

\begin{tabular}{lllll}
\hline & \multicolumn{2}{c}{$\alpha=0, \beta=0.1$} & \multicolumn{2}{c}{$\alpha=0.1, \beta=0.1$} \\
& $r_{\mathrm{d}}=0$ & $r_{\mathrm{d}}=0.5$ & $r_{\mathrm{d}}=0$ & $r_{\mathrm{d}}=0.5$ \\
\hline$\gamma=0.0$ & 1.7967 & 0.8524 & 1.3287 & 0.7434 \\
$\gamma=0.1$ & 1.1481 & 0.5936 & 0.8995 & 0.5131 \\
$\gamma=0.2$ & 0.7054 & 0.3686 & 0.5718 & 0.3112 \\
$\gamma=0.3$ & 0.3923 & 0.1795 & 0.3166 & 0.1398 \\
\hline
\end{tabular}

Table 5: Bound $r_{M}$ for $r_{d}=0.1$ and $\tau_{d}=0$ and various values of the parameter $c$

\begin{tabular}{|c|c|c|c|c|c|c|c|c|}
\hline$|c|$ & 0.0 & 0.1 & 0.2 & 0.3 & 0.4 & 0.5 & 0.6 & 0.7 \\
\hline$r_{\mathrm{M}}$ & 0.97 & 0.78 & 0.60 & 0.45 & 0.31 & 0.19 & 0.10 & 0.02 \\
\hline
\end{tabular}

Case I: For $c \equiv 0$ and $\boldsymbol{h}(\dot{\boldsymbol{x}}(t-\tau(t)), t) \equiv 0$, the system under consideration reduces to the system studied in [13]. Applying criteria in [13], [15] and in this work, the maximum value of $r_{M}$ for the stability of the system is listed in Table 1. It is easy to see that our proposed stability criterion gives a much less conservative result than one in [13] and [15]. Other results surveyed in [13] are even more conservative.

Case II: For $\boldsymbol{h}(\dot{\boldsymbol{x}}(t-\tau(t)), t) \equiv 0$ and $\tau_{\mathrm{d}}=0$, the maximum value $r_{\mathrm{M}}$ is listed in Table 2 for different $c$. As $|c|$ increases, $r_{\mathrm{M}}$ decreases.

Case III: For $c=0.1$ and/or $\tau_{\mathrm{d}}=0\left(\tau_{\mathrm{d}}=0.5\right)$, we now consider the effect of an uncertainty bound $\gamma$ on the maximum value $r_{\mathrm{M}}$. Tables 3 and 4 illustrates the numerical results for different $\gamma, \tau_{\mathrm{d}} \equiv 0$ and $\tau_{\mathrm{d}}=0.5$, respectively. We can see that $r_{\mathrm{M}}$ decreases as $\gamma$ increases.

Example 3: Consider the following uncertain linear neutral system with a time-varying discrete delay:

$$
\begin{aligned}
\dot{\boldsymbol{x}}(t)= & \left(\begin{array}{cc}
-2+\delta_{1} & 0 \\
0 & -1+\delta_{2}
\end{array}\right) \boldsymbol{x}(t) \\
& +\left(\begin{array}{cc}
-1+\delta_{3} & 0 \\
-1 & -1+\delta_{4}
\end{array}\right) \boldsymbol{x}(t-r(t)) \\
& +\left(\begin{array}{ll}
c & 0 \\
0 & c
\end{array}\right) \dot{\boldsymbol{x}}(t-\tau(t))
\end{aligned}
$$

where $0 \leq|c|<1$ and $\delta_{1}, \delta_{2}, \delta_{3}$ and $\delta_{4}$ are unknown parameters satisfying:

$$
\left|\delta_{1}\right| \leq 1.6 \quad\left|\delta_{2}\right| \leq 0.05 \quad\left|\delta_{3}\right| \leq 0.1 \text { and }\left|\delta_{4}\right| \leq 0.3
$$

Case I: For $c=0$ and $r_{\mathrm{d}} \equiv 0$, applying criteria in [10] and [23], the maximum value of $r_{\mathrm{M}}$ for stability of system (29) is 0.2412 and 1.0, respectively. By proposition 3, it is found that the maximum value of $r_{\mathrm{M}}$ is 1.0345 .

Case II: For $r_{\mathrm{d}}=0.1$ and $\tau_{\mathrm{d}}=0$, the maximum value of $r_{\mathrm{M}}$ is listed in Table 5 for various values at the parameter $c$. As $|c|$ increases, $r_{\mathrm{M}}$ decreases.

Case III: For $c=0.1$ and $\tau_{\mathrm{d}}=0$, we obtain the maximum value of $r_{\mathrm{M}}$ in Table 6 . One can see that as $r_{\mathrm{d}}$ increases, $r_{\mathrm{M}}$ decreases.

Case IV: For $c=0.1, r_{\mathrm{d}}=0.1$, the effect of $\tau_{\mathrm{d}}$ on the

\begin{tabular}{|c|c|c|c|c|c|c|c|c|c|c|}
\hline$r_{\mathrm{d}}$ & 0.0 & 0.1 & 0.2 & 0.3 & 0.4 & 0.5 & 0.6 & 0.7 & 0.8 & 0.9 \\
\hline$r_{\mathrm{M}}$ & 0.82 & 0.78 & 0.74 & 0.69 & 0.63 & 0.57 & 0.50 & 0.42 & 0.32 & 0.17 \\
\hline
\end{tabular}
maximum value $r_{M}$ is listed in Table 7 . One also sees that as $\tau_{\mathrm{d}}$ increases, $r_{\mathrm{M}}$ decreases.

\begin{tabular}{|c|c|c|c|c|c|c|c|c|c|c|}
\hline$\tau_{\mathrm{d}}$ & 0.0 & 0.1 & 0.2 & 0.3 & 0.4 & 0.5 & 0.6 & 0.7 & 0.8 & 0.9 \\
\hline$r_{\mathrm{M}}$ & 0.78 & 0.77 & 0.76 & 0.74 & 0.73 & 0.70 & 0.68 & 0.63 & 0.57 & 0.42 \\
\hline
\end{tabular}

Table 6: Bound $r_{M}$ for $c=0.1$ and $\tau_{d}=0$, and different values for $r_{d}$

Table 7: Bound $r_{M}$ for $c=0.1$ and $r_{d}=0.1$, and different values of $\tau_{d}$ 


\section{Conclusions}

The robust stability problem of uncertain linear systems with time-varying discrete and neutral delays has been studied. Some practically computable stability criteria have been obtained. The results have included some existing results as their special cases. Examples have also been given to show significant improvements over existing results in the literature.

\section{Acknowledgments}

The research work of Q.-L. Han was supported in part by Central Queensland University for the 2004 Research Advancement Awards Scheme Project 'Analysis and Synthesis of Networked Control Systems'. The research work of $\mathrm{L}$. $\mathrm{Yu}$ was supported by the National Natural Science Foundation of China under grant 60274034.

\section{References}

1 Hale, J.K., and Verduyn Lunel, S.M.: 'Introduction to functional differential equations' (Springer-Verlag, New York, 1993)

2 Brayton, R.K.: 'Bifurcation of periodic solutions in a nonlinear difference-differential equation of neutral type', Q. Appl. Math., 1966, 24, pp. 215-224

3 Kuang, Y.: 'Delay differential equations with applications in population dynamics', in 'Mathemathics in Science and Enginering' (Academic Press, San Diego, CA, 1993), vol. 191

4 Slemrod, M., and Infante, E.F.: 'Asymptotic stability criteria for linear systems of differential equations of neutral type and their discrete analogues', J. Math. Anal. Appl., 1972, 38, pp. 399-415

5 Verriest, E.-I., and Niculescu, S.-I.: 'Delay-independent stability of linear neutral systems: a Riccati equation approach', in Dugard, L., and Verriest, E.I. (Eds.): 'Stability and control of time-delay systems' (Springer-Verlag, London, 1997), pp. 92-100

6 Han, Q.-L.: 'On delay-dependent stability for neutral delay-differential systems', Int. J. Appl. Math. Comput. Sci., 2001, 11, pp. 965-976
7 Han, Q.-L.: 'Robust stability of uncertain delay-differential systems of neutral type', Automatica, 2002, 38, pp. 719-723

8 Niculescu, S.-I.: 'On delay-dependent stability under model transformations of some neutral linear systems', Int. J. Control, 2001, 74, pp. 609-617

9 Fridman, E.: 'New Lyapunov-Krasovskii functionals for stability of linear retarded and neutral type systems', Syst. Control Lett., 2001, 43 pp. 309-319

10 Fridman, E., and Shaked, U.: 'Delay-dependent stability and $H_{\infty}$ control: constant and time-varying delays', Int. J. Control, 2003, 76, pp. $48-60$

11 Wang, S.S., Chen, B.S., and Lin, T.P.: 'Robust stability of uncertain time-delay systems', Int. J. Control, 1987, 46, pp. 963-976

12 Goubet-Batholomeus, A., Dambrine, M., and Richard, J.P.: 'Stability of perturbed systems with time-varying delays', Syst. Control Lett., 1997, 31, pp. $155-163$

13 Cao, Y.-Y., and Lam, J.: 'Computation of robust stability bounds for time-delay systems with nonlinear time-varying perturbations', Int J. Syst. Sci., 2000, 31, pp. 359-365

14 Kharitonov, V.L., and Melchor-Aguilar, D.: 'Additional dynamics for time-varying systems with delay'. Proc. 40th IEEE Conf. on Decision and Control, 2001, pp. 4721-4726

15 Han, Q.-L.: 'On robust stability for a class of linear systems with timevarying delay and nonlinear perturbations', Comput. Math. Appl.An International Journal, 2004, 47, pp. 1201-1209

16 Park, P.: 'A delay-dependent stability criterion for systems with uncertain time-invariant delays', IEEE Trans. Autom. Control, 1999 44, pp. 876-877

17 Boyd, S., Ghaoui, L.El., Feron, E., and Balakrishnan, V.: 'Linear matrix inequalities in systems and control theory', Stud. Appl. Math., 1994, 15

18 Kolmanovskii, V.B., and Myshkis, A.: 'Applied theory of functional differential equations' (Kluwer Academic Publishers, Boston, MA 1992), p. 129

19 Yakubovich, V.A.: 'S $\mathcal{S}$-procedure in nonlinear control theory', Vestn. Leningr. Univ. 1, Mat. Mekh., 1971, 13, pp. 62-77

$20 \mathrm{Li}, \mathrm{X}$., and de Souza, C.E.: 'Criteria for robust stability and stabilization of uncertain linear systems with state delay', Automatica, 1997, 33 pp. $1657-1662$

21 Moon, Y.S., Park, P., Kwon, W.H., and Lee, Y.S.: 'Delay-dependent robust stabilization of uncertain state-delayed systems', Int. J. Control, 2001, 74, pp. 1447-1455

22 Gahinet, P., Nemirovski, A., Laub, A.J., and Chilali, M.: 'LMI contro toolbox: for use with MATLAB' (Math Works, Natick, MA, 1995)

$23 \mathrm{Kim}$, J.-H.: 'Delay and its time-derivative dependent robust stability of time-delayed linear systems with uncertainty', IEEE Trans. Autom. Control, 2001, 46, pp. 789-792 